\title{
Geriatric dermatology, changes related to aging
}

\author{
Israel Sánchez-Álvarez ${ }^{1 *}, M^{a}$ Ivonne Arellano-Mendoza ${ }^{1}$ and Lorenzo García ${ }^{2}$ \\ ${ }^{1}$ Servicio de Dermatología; ${ }^{2}$ Servicio de Geriatría. Hospital General de México, Mexico City, Mexico
}

\begin{abstract}
Due to the population growth in our country, it is extremely important to disclose the different medical specialties that cater to primary care and treatment, in addition to those related to secondary care. Many are the skin diseases that mostly affect elderly people; hence, we summarize the most common ones and those diseases causing the greatest impact on life quality. The present study aims to provide an approach to the physiological changes and main characteristics of the skin of elderly people; furthermore, it lists the main benign skin conditions whose diagnosis represents a great deal of intervention in these patients. The most prevalent bullous diseases and neoplasms have also been added. We conclude that the Mexican primary health-care sector needs more in-depth training about the changes in the elderly population and their main skin diseases.
\end{abstract}

Key words: Geriatric dermatology. Geriatrics. Elderly skin. Age related skin changes. Skin diseases in geriatrics.

\section{Introduction}

The growth of elderly population over the age of 60 in Mexico has been on an upward trend since 1990. According to the National Institute of Statistics and Geography records in its 2010 population and housing census, the aging index was 31 elderly adults per 100 children aged 14 years, demonstrating the reversed trend of the population pyramid ${ }^{1}$. According to the WHO, most countries of the developed world have accepted the chronological age of 65 years as a definition of "elders" or elderly people. Although the United Nations has no officially accepted definition, the age of 60 years is generally accepted as referring to old age or senior patients or simply elderly patients ${ }^{2}$. Under these conditions, the population growth of this age group in Mexico is accompanied by an increase in chronic diseases and greater physiological vulnerability, which makes their evaluation and management much more complex, requiring greater demand for care and consumption of resources. Thus, it is necessary to identify needs, detect risks, and provides specialized care in this age group ${ }^{3}$.

Eldercare requires a comprehensive evaluation, with emphasis on everyday activities, functionality, psychological conceptions, personality, behavior, family dynamics, and socioeconomic conditions of senior citizens; all these influence interactions between them and their health condition, requiring multidisciplinary assistance with care models based on interdisciplinary evaluation as proposed by Wong and Koo, from the University of San Francisco, California, who have named it "Dermatogeriatrics." specialized human resources in this area, they are still insufficient to meet the needs of this group. Hence, it is necessary to inform primary health-care professionals about the aging processes that affect the skin so that they can identify clinical conditions and associated

\section{Correspondence:}

*Israel Sánchez-Álvarez

E-mail: dr.israelsanchez@gmail.com
Available online: 13-09-2019

Date of reception: 05-06-2017

Date of acceptance: 27-07-2017

DOI: 10.24875/HGMX.M19000026
Rev Med Hosp Gen Mex. 2019;82(3):158-168

www.hospitalgeneral.mx

0185-1063/@ 2017 Sociedad Médica del Hospital General de Mexico. Published by Permanyer México SA de CV. This is an open access article under the CC BY-NC-ND license (http://creativecommons.org/licenses/by-nc-nd/4.0/). 
comorbidities in a practical way, to perform interventions or refer these patients to medical specialists in a timely manner to maintain and recover functionality.

\section{Materials and methods}

A systematic search was conducted in the PubMed database of the literature available from 2000 to 2016 with the words: skin diseases, skin aging, skin neoplasms, skin diseases, Vesiculobullous, skin changes, elderly, skin diseases metabolic, skin manifestations, skin physiological, phenomena, and aged. In total, forty articles were found with different information. Due to the broad scope of the topic, the main topics were reviewed highlighting the most important diseases and impact on the morbidity and mortality of patients, focusing on the changes associated with the aging process, the main benign dermatoses, bullous diseases, and most common neoplasms affecting this age group.

\section{Development and discussion}

\section{Physiological changes associated with skin aging}

The skin is a complex organ, exposed to multiple external and internal aggressions. It is seen as a shock organ that can be affected by a wide variety of immunological, endocrine, neuropsychiatric, metabolic, and tumor aspects, among others. Roughly speaking, the skin consists of three complex layers with multiple functions.

The epidermis is the first and outermost layer, composed of a keratinized stratified epithelium with important protective functions, such as melanogenesis, which protects epidermal cells from damage caused by UV radiation. It also has metabolic and immunological functions as evidenced by studies on Vitamin D with an important autocrine function involved in the differentiation and proliferation of the keratinocyte. Moreover, it plays an important role in the development of neoplasms, by promoting apoptosis and inducing the transcription of several proapoptotic genes (BAX, Bcl-2, $F A D$, and $C F K A R)^{5}$. Its multiple immunological functions act as a barrier in our interactions with the external environment, containing various mechanoreceptors, and nociceptors such as Merkel cells, and Pacini and Krause corpuscles where a wide variety of physical and chemical stimuli are received ${ }^{6,7}$.

The basement membrane separates the epidermis from the dermis. Its primary function is to anchor down the epidermis. The different molecules that make up this assembly are the target of various autoantibodies, key in the etiopathogenesis of bullous diseases ${ }^{6,7}$.

The dermis is composed of a fundamental substance formed mostly by proteoglycans and glycosaminoglycans, which act as adhesion molecules for collagen fibers, which in turn provide the supportive connective tissue, by protecting the various structures from mechanical damage. On the other hand, the elastic fibers grant deformation resistance. The production of this extracellular matrix consists of fibroblasts, which have a fundamental role in the epithelial-mesenchymal interaction while producing various cytokines, growth factors, and differentiation. It also has a large part of the annexes composed of the pilosebaceous unit, eccrine, apocrine, and sebaceous glands with important functions such as moisturizing, hydration, and $\mathrm{pH}^{6,7}$.

Finally, the subcutaneous tissue is composed of adipocytes arranged in lobules, separated by fibrous tissue septa. Its primary function is as an insulator to help regulate body temperature, energy storage, and buffering. Metabolic functions are currently recognized; leptin and adiponectin are produced related to the regulation of appetite; it produces proinflammatory cytokines such as tumor necrosis factor-alpha (TNF- $\alpha$ ), and interleukin (IL) 1 and 6 , has vascular activity by regulating the production of angiotensin and inhibitor of plasminogen activator type 1, besides playing an important role in the development of insulin resistance by means of resistin ${ }^{6,7}$.

\section{Physiological skin aging}

Two types of aging can be considered, intrinsic aging related to the natural chronoaging process and extrinsic or photoaging related to the damage induced by ultraviolet radiation $(\mathrm{UVR})^{8}$. All functions are reduced during the aging process. In addition to decreasing in metabolic, mitotic and immune activity, production of extracellular matrix is reduced considerably, with decrease of collagen fibers I and III, fatty acid stores are reduced; immunological activity is altered, with a tendency toward the production of proinflammatory cytokines such as IL-16, interferon-gamma, and TNF- $\alpha$, producing a state of systemic inflammation known as "inflammaging." The oxidative damage produced by the accumulation of free radicals increases the levels of reactive oxygen species adding to the inflammatory damage, the action of ultraviolet radiation induces changes not only in the nucleolar machinery but also mutations detected in the main cutaneous neoplasms, 
at the level of the dermis, further deteriorating the repair process ${ }^{9,10}$. In women, the decrease in estrogen levels accelerates the aging process. The hair becomes less thick and the melanocytic activity of the hair follicle is lost. Thinning of axillary and pubic hair takes place because of the decrease in circulating androgen levels. The nail plate of the fingers suffers progressive thinning (onychorrhexis), while the plates of the toenails undergo thickening (pachyonychia), sometimes producing deformations of the entire plate (onychogryphosis), also secondary to the deformity of the osseous structures with the consequent alteration of the gait. In addition, the pigmentation of the nail plate secondary due to friction (frictional melanonychia). Conversely, there is a decrease in the number of salivary glands in the oral mucosa, reducing the amount and quality of saliva that results in changes affecting the taste, chewing, digestion, and articulation of words, also increasing the incidence of opportunistic infections such as oral candidiasis, bacterial infections, cavities, and periodontal disease ${ }^{8}$. In general, many changes occur due to the natural aging process, besides changes produced by individual comorbidities.

\section{Changes associated with physiological skin aging}

There are several changes associated with the multiple alterations suffered by the skin in the natural aging process such as the decrease in function at all levels that lead to important changes in the cutaneous homeostasis of these individuals. It begins with a decrease in the DNA repair capacity; the keratinocyte presents mutations induced mainly by photocarcinogenesis giving rise to the development of neoplasms. The reduced production of sebum and sweat by the sebaceous glands, together with a lower cell turnover rate, favor dryness and roughness of the skin, as well as delay in the repair of tissues. Fragmentation of collagen and elastic fibers in the dermis promotes skin laxity and wrinkle formation; the vascular plexus diminishes, causing the appearance of ecchymosis and purple spots (senile purpura). Reduced sensory perception translates as a decrease to external stimuli facilitating injuries and making for higher risk of mechanical and chemical thermal injuries. Androgenic hormonal interaction produces changes at the hair level stimulating the growth of hair in places like the auricles that is mainly due to the holandric gene inheritance, and although it usually appears after the age of 45 years, this is observed in this population. The increase of the anagen phases of eyebrows and nasal hair along with miniaturization of the terminal hairs on the scalp, determine the appearance of androgenetic alopecia in men; while in women, terminal hairs appear in facial areas generally associated with male hair growth such as the beard and mustache zone. In addition, the decrease in melanocytes directly affects hair pigmentation, causing the appearance of gray hair at different levels; likewise, fatty acid stores are affected, presenting a thinning of this supportive connective, storage and insulation tissue, which increases the risk of hypothermia and enhances the mechanical forces that can damage the tissues. Due to these series of changes, the organism of elderly people becomes fragile, minimizing its response and repair capacity so that homeostasis is easily lost, which takes a toll on their quality of life $\mathrm{e}^{8,11}$.

\section{Xeroderma}

Xeroderma is one of the main dermatosis of the elderly population, with pruritus being associated as the main symptom; the impact on the quality of life is relevant and maybe the cause of secondary infections; clinically, it is identified by the presence of blood crusts, abrasions and lichenification zones that settle on a dry and fragile skin. The pathogenesis is complex, but it can be mainly considered that skin aging with the consequent decrease in cell turnover rate, lipid deficiency, and loss of aquaporins (AQP3) leads to alteration between the balance of moisture and hydration. The $\mathrm{pH}$ tends to be more alkaline, altering the enzymatic activity with the consequent decrease in the lipids contained in the lamellar bodies. In addition to the activation of the serine proteases, involved in the physiological desquamation of the stratum corneum and the protease receptor (PAR2), which directly causes the pruritus.

The nervous degeneration secondary to diabetes mellitus (DM) is another important factor ${ }^{12}$; the damage produced by herpes zoster at the ganglionic level has also been implied, producing post-herpetic pruritus in up to $36 \%$ of the cases, other localized pruritus mechanisms are related to radiculopathies, as in notalgia paresthetica $^{13}$.

According to data from the Pramukhswami Medical College in India, it is associated with multiple comorbidities such as DM, which is considered one of the cardinal skin disease symptoms. Another associated pathology is hyperthyroidism which is deemed to be related to antithyroid antibodies, although the cause remains uncertain, it has been associated with the 
increase in temperature and water retention of the skin of these patients. In the case of chronic kidney disease, the highest prevalence is observed in $60-90 \%$ of uremic patients in dialysis treatment, the cause is related to alterations in the balance of sodium, phosphorus, and magnesium, in addition to the release of proinflammatory cytokines and histamine ${ }^{12}$. Other systemic disorders include hypothyroidism, iron deficiency anemia, polycythemia, lymphoproliferative and myeloproliferative disorders, atopic dermatitis and ichthyosis, lichen simplex chronicus (LSC), cutaneous infections, psoriasis, hives, insect bite prurigo, and drug-induced skin reactions, among others ${ }^{13}$.

The diagnosis must be thorough, ruling out other causes of secondary pruritus that may have greater impact such as scabies, chronic idiopathic urticaria, and systemic diseases such as hematological neoplasms, hepatobiliary disorders, and polypharmacy in the presence of comorbidities (ACE inhibitors, thiazides, salicylates, codeine, and antimalarial medications). The physical examination must be comprehensive, ruling out other dermatological causes of the pruritus; laboratories should be instructed in accordance with the underlying condition or suspicion, complete blood count, liver function tests, tumor markers, and imaging studies should be performed in cases of a suspected underlying neoplasm ${ }^{12}$.

Treatment should be aimed to improve general skin conditions, the use of topical emollients and soap substitutes is of great help ${ }^{13}$; baths should be short and with warm water in order not to decrease the lipid layer that provides moisture to the skin. Urea-containing creams function as a natural humectant factor; topical steroids are reserved for cases of itching where inflammation is present, as their multiple side effects must be taken into account, and long-term use should be avoided. Regarding systemic treatment with first- and second-generation antihistamines, first-generation antihistamines are indicated for nocturnal pruritus because of their sedative effects, and second-generation antihistamines are indicated for histaminergic disorders such as chronic urticaria. Conversely, side effects should be assessed, such as drowsiness and urinary retention ${ }^{14}$. There are a number of reports and cases related to multiple antidepressants, which present several adverse effects, so they are restricted to certain groups, in particular, thalidomide used in refractory pruritus, which owes its antipruritic effects to the involvement of the $C$ fibers in the neural pathways of pruritus, in addition to its immunomodulatory effects. When therapeutic possibilities have been exhausted, or none of them are viable, narrowband ultraviolet B (NB-UVB) phototherapy represents a therapeutic option while producing mast cell apoptosis and inhibiting the Th1 response. The risk-benefit ratio in Caucasian patients must always be assessed because of the risks associated with the development of melanoma and basal cell carcinomas. Acupuncture and psychobehavioral therapy has shown some degree of effectiveness, but lack scientific evidence ${ }^{12-14}$.

\section{Atopic dermatitis in adults}

Atopic dermatitis is a multifactorial, chronic, and relapsing-remitting disease with multiple clinical spectra, but with similar characteristics. In the case of adults, it mainly affects flexion and extension zones, such as antecubital and popliteal fossae, although it can affect all body segments or become generalized (erythroderma). Clinically speaking, it is characterized by erythematous-based plaques covered by a thin whitish scale that, together with the chronicity of the condition, tends to develop into lichenification, blood crusts suggestive of active scratching, and hypochromic and hyperchromic spots in areas of remission. Some classic observable signs consist of Dennie-Morgan's double fold, located on the lower eyelid, and the sign of Hertoghe (loss of the outer third of eyebrows), among others ${ }^{6}$.

The etiology is uncertain; multiple mechanisms have been implicated such as "immunosenescence" secondary to thymic involution, reduced population of Naive $T$ lymphocytes, and increased number of memory $T$ lymphocytes, another added factor is the decrease in androgen levels and its impact at the cutaneous and immunological level. The loss of cutaneous and intestinal barriers is associated with greater susceptibility to environmental irritants and allergens ${ }^{15}$.

According to the serological data, it can be classified as IgE-mediated, which has high titers of up to $400 \mathrm{IU} / \mathrm{L}$, positive epicutaneous tests and sensitivity to environmental antigens, mainly mites Dermatophagoides sp. and pollen. The non-lgE variety presents normal titers and lack of sensitization to environmental antigens, with a predominance of the former in up to $63.3 \%{ }^{15}$. The course of this disease can be de novo (geriatric onset), recurrent, with onset in childhood and recurrent in adults, with a history of atopic march since childhood, outbreaks in adolescence and adulthood. Complications are usually secondary infections due to barrier disruption and asthmatic symptoms associated with elevated IgE levels ${ }^{15}$.

Treatment is based on the basic pathology, controlling the symptoms, improving the quality of life, emollients, 
topical steroids, and calcineurin inhibitors (tacrolimus and pimecrolimus), the control of pruritus can be managed with antihistamines, assessing the risk-benefit ratio due to the possibility of patients developing delirium or slow transit constipation ${ }^{14,15}$. Systemic steroids are reserved for widespread or recalcitrant cases in relation to conservative treatment. Prednisone is generally used at anti-inflammatory doses $(<0.5 \mathrm{mg} / \mathrm{kg})$ and should be used with caution because of its multiple side effects and concomitant polypharmacy. Cyclosporine has been used instead with excellent results but with important adverse effects such as nephrotoxicity and the development of neoplasms. The use of NB-UVB phototherapy at a dose of $0.35-0.65 \mathrm{~J} / \mathrm{cm}^{2}$ per session has also yielded excellent results but has a risk associated with the development of malignant neoplasms ${ }^{15}$.

\section{Dermatoporosis}

Also known as chronic cutaneous insufficiency, this term was described by Saurat et al. in $2007^{16}$ and is characterized by a progressive loss of the constitutive elements of the skin appearing after the age of 70 years ${ }^{17}$. Other sources report its appearance after the age of 60 years affecting 1 in 3 individuals ${ }^{18}$, according to a French study in which 202 patients aged 60-80 years were evaluated, showing prevalence in $32 \%$ of patients with a predominance in men at $38 \%{ }^{17}$. As aforementioned, the skin of elderly patients presents a series of changes and physiological events that are typical of the degenerative process that leads to aging at all levels. In particular, the skin presents hyaluronic acid (HA) loss which is one of its main components whose function is based on stabilizing the cutaneous structures by forming a series of bridges between collagen and elastic fibers, forming a viscous network that protects skin against the mechanical aggressions of the environment ${ }^{16}$. This reduced HA synthesis is due to the suppression of CD44 positive keratinocytes which bind these glycosaminoglycans and whose expression is diminished in patients with dermatoporosis ${ }^{17}$. This decrease leads to a series of changes that are clinically reflected as skin fragility and because the topography mainly occurs in photo-exposed areas. Elastosis is believed to play an important role in the development of said pathology. Studies with animal models demonstrate the loss of CD44 and $\mathrm{AH}$ expression in keratinocytes of UVB-irradiated mice also activating metalloproteinases.

Clinically, it is characterized by affecting photo-exposed areas such as forearms, the back of the hands, the anterior side of legs in the pretibial area and " $\mathrm{V}$ " of the neckline. It mainly consists of cutaneous atrophy; the skin becomes thinner, fragile, and shiny. Ultrasounds show a decrease in the total thickness of epidermis and dermis from $0.7 \mathrm{~mm}$ to $0.8 \mathrm{~mm}$. Purple spots (senile purpura) appear spontaneously even in patients without anticoagulant treatment. Most patients develop hemosiderin staining giving the skin an ochre color. Pseudo-stellate scars are formed because of the diminished proliferation of fibroblasts and increase of local metalloproteinases. Histologically, they correspond to compact hypocellular collagen bands with mucin deposits and severe elastolysis. Dissecting hematomas can be presented according to the disease stage such as accumulation of blood in a third space up to necrotic plaques because of the vascular compression exerted by the volume thereof and "SCALP" type lacerations produced by shear forces with minor injuries. All these characteristics are also considered morphological markers that are staged in different degrees ranging from I to IV ${ }^{16,18}$. It is classified as primary or pathological dermatoporosis when it is believed to be influenced by genetic factors, and as secondary, when it is believed to be due to the use of corticosteroids that accentuate atrophy ${ }^{16-18}$.

Treatment is based on prevention with the use of sunscreens and emollients that provide adequate moisture and promote hydration. However, on the onset of the problem, the approach will depend on which stage it is at, by always evaluating additional comorbidities and dermatosis. Action protocols vary, among them, treatments with paraffin oil and lanolin-based emollients in Stages I to III. Dissecting hematomas and necrotic plaques should be treated in specialized hospitals and treatment is based on hydrocolloid dressings, alginates, and antibiotics, in the case of infections. It is now known that retinoids are an effective treatment inducing the expression of GFT $\beta$, the expression of CD44 stimulating the keratinocyte proliferation process, and increasing collagen production. Other treatments, such as topical intermediate-sized HA fragments (HAFi), have been shown to restore the expression of CD44 and GFT $\beta$, by promoting the restoration of the epidermal barrier ${ }^{16,18}$.

\section{Benign pigmented skin lesions and photoaging}

The accumulation of ultraviolet radiation on the skin produces molecular lesions at the cellular DNA level causing mutations and degenerative changes on the dermis degenerating collagen and elastic fibers. These changes are more accentuated in populations with 
phototypes I to III in whom the melanin protection is not as marked as in phototype IV to VI populations ${ }^{9}$.

These changes occur mainly in photo-exposed areas such as the face, cleavage, arms, and hands, commonly affecting populations whose labor activity mainly occurs outdoors. In a study published in the International Journal of Dermatology, where 198 subjects $\geq 65$ years old were evaluated, a high prevalence of benign pigmented skin lesions was found, such as solar lentigines and seborrheic keratosis that were not only related to photocarcinogenic, chrono, and photoaging processes. Furthermore, it is related to the presence of human papillomavirus (HPV) because of the mutation of the $\mathrm{FGRF}_{3}$ gene $^{19}$. These lesions are directly related to age, their correct identification implies prognostic factors and treatment, as in the case of lentigo maligna melanoma. Thus, the presence of actinic damage associated with a pigmented skin lesion with atypical clinical features is a warning of a malignant melanocytic lesion, which should be strongly suspected if there are growth data (presence of peripheral pigment globules) in patients older than 50 years old ${ }^{19}$.

The appearance of pigmented skin lesions is mainly due to a phenomenon called nevi genesis, with two potential hypotheses, the first one called constitutional or congenital hypothesis, which is related to peripheral globular structures, is derived from melanoblasts. Clinically, it presents itself as an exophytic, cupuliform or hemispherical neoformation, with regular edges, symmetrically shaped and having a homogeneous pigment. On the other hand, the second case, called acquired or exogenous hypothesis presents a peripheral halo of small globular structures, derived from intra-epidermal melanocytes, which proliferate by stimuli such as UV radiation ${ }^{19}$. Hence, the presence of a poorly delimited, flat, hyper-pigmented brown macular neoformation with heterogeneous pigment should be referred to a specialist for evaluation in the case of a lentigo malignant melanoma.

\section{Solar lentigines}

Also known as lentigo senilis and age spots, they are among the main dermatoses in elderly age ${ }^{10}$. These are located in areas that are vulnerable to damage due to sun exposure such as the face, neck, neckline, forearms, and the back of the hands. They are described as macular neoformations with variable size and hyper-pigmented, ranging from light to dark brown colors. Histologically, elongation, and fusion of the interpapillary processes are observed with an increase in the number of melanocytes and melanin. Elastosis can be observed in the dermis ${ }^{20}$.

Several growth factors and their receptors produced by fibroblasts with paracrine and melanogenic action directly related to UV exposure have been involved in its development. In a study conducted in the cell biology laboratory of the National Cancer Institute, twenty Caucasian subjects with phototypes I and II between the ages of 55 and 73 years were evaluated. A biopsy of solar lentigines was performed with specimens analyzed by immunohistochemical techniques and microarray analysis. A relative increase in the density of the melanocytes was observed but without an increase in the epidermal thickness and density. Conversely, the DNA microarray analysis showed that there is no specific gene expression pattern for the different melanocytes in perilesional skin. Therefore, it is suggested that the formation of solar lentigines is not related to the increase of melanin or the number of melanocytes, but their decreased metabolic activity. In this same study, an alteration between the expression of cytokeratins KRT15 and KRT10 was also observed; showing the alteration that basal and suprabasal keratinocytes suffer, consisting in the slowdown of the scaling process of the latter, with an increase in the proliferation of the former, which leads to pigment accumulation ${ }^{21}$.

First, they should be distinguished from malignant pigmented skin lesions due to the morbidity and mortality represented by the latter. Treatment is varied, cryotherapy with liquid nitrogen, chemical peels based on trichloroacetic acid or laser Q-Switched ND: YAG and $\mathrm{CO}_{2}$ can be used. Because they do not pose any significant risks, these treatments are merely aesthetic; although if this represents a problem of perception related to well-being, they should be treated in a correct way in relation to the Biopsychosocial context of the individual ${ }^{14,20,21}$.

\section{Seborrheic keratosis}

Seborrheic keratoses are benign tumors of epithelial origin. They are also known as senile wart, seborrheic wart, and basal cell acanthoma, among others. Its clinical presentation is varied considering five variants or types: common, dermatosis papulosa nigra, pediculated, flat, and stucco. Clinically, they appear as circular or oval neoformations, whose size ranges from millimeters to several centimeters and are hyper-pigmented with a light to dark brown color. Although they can appear unpigmented as in lighter phototypes, their surface is unctuous, velvety with a warty appearance. 
They appear mainly on the face, neck, trunk, "V" area of the neckline, upper limbs, on the back of hands, forearms, and arms, although they can appear in any part of the body, as there are case reports of seborrheic keratosis in the conjunctiva and mucosa. Its prevalence is directly proportional to the age of the individual, more frequently appearing after the age of 50 years, affecting both sexes equally and is observed mostly in Caucasian populations.

Its pathogenesis is uncertain, involving several factors such as cumulative exposure to UV radiation, which increases its incidence up to 2.3 times and this is believed as the cause of mutations in the FGRFR3 receptor, which would cause a monoclonal proliferation of basaloid cells. Histologically speaking, they are well-differentiated tumors, composed of basaloid-like keratinocytes that anastomose in the form of strands, leading to acanthosis, and papillomatosis. It is common to observe keratin accumulations forming corneal pseudocysts; histological varieties have been described as solid, adenoid, papillomatous, clonal, and irritated ${ }^{24}$. Association with neoplasms has been reported, by forming paraneoplastic syndromes such as LeserTrelat, related to gastrointestinal tumors. It presents itself as multiple eruptive seborrheic keratoses in a short period of time ${ }^{6}$.

Diagnosis is essentially clinical, and dermatoscopy is shown to be significantly helpful. It allows us to discriminate between malignant pigmented skin lesions and collision tumors; the main structures observed are pseudocysts and follicular openings. A differential diagnosis is important as errors lead to important morbidity, especially in melanocytic lesions, such as the case of verrucous melanoma, pigmented basal cell carcinoma, and melanocytic nevi ${ }^{24}$.

Treatment is based on the removal of the tumor by curettage, shaving, electrodessication, and cryotherapy. Some more sophisticated methods include Er: YAG laser or $\mathrm{CO}_{2}$ laser with good esthetic results in general, and the prognosis is good for benign tumors ${ }^{25}$.

\section{Nail abnormalities}

The nail is a complex specialized organ with diverse functions that include protection, fine touch, scratching, and even defense. It consists of a nail plate, known as the nail itself. It is composed of compact keratin and sulfate amino acids that give to it its rigidity. The nail matrix is responsible for the production of the nail; the bed is responsible for providing support and forms a minimum portion of the nail plate and lateral, proximal and distal folds in charge of insulating and protecting the structures from external agents ${ }^{26}$. Nail alterations can occur at any age; however, major changes occur in adults, including those associated with the physiological process of aging and abnormalities intrinsic to the nail itself, and those secondary to other dermatoses, systemic diseases, physical, and chemical factors. Due to the increase in life expectancy, there is also an increase in the frequency of multiple abnormalities that can lead to complications and adverse effects associated with the treatment thereof ${ }^{27}$.

Among the physiological changes of aging, changes occur in the thickness, reduced growth, and various changes in coloration. Particularly in the fingernails, there is a thinning of the plate accompanied by retraction of eponychium and formation of longitudinal ridges, nail fragility with horizontal splits (onychoschisis) mostly occurring in $36 \%$ of females aged $\geq 60$ years probably due to hormonal stimulation and contact with chemicals, detergents, solvents, and cosmetics ${ }^{25}$. The most relevant changes occur on the toenails, most of them associated with a continuous injury process, due to the biomechanical alterations of the foot (hallux valgus) causing thickening of the nail plate (pachyonychia) and of the lateral and subungual folds which leads to the formation of ingrown toenails (onychocryptosis) and subungual tylosis (onychoclavus). The constant friction of the toes with the shoes in darker phototypes leads to the pigmentation of the nail plate (frictional melanonychia) or may not be related to the frictional stimulus (xanthonychia). In addition, limited ranges of motion and presbyopia prevent nail trimming, causing deformity of the nail plate in the form of "ram's horns" (onychogryphosis), among the main physiological alterations arising from aging ${ }^{7}, 28$.

\section{Onychomycosis}

The prevalence of onychomycosis is important, reaching percentages that reach $50 \%$ according to the population studied. In a multicentric study conducted in 2010 in Mexico, 12,637 people were interviewed, consisting of a survey with 17 questions prepared by the Asociación Mexicana de Micología Médica AC (Mexican Association of Medical Mycology AC). It has clinically diagnosed $48 \%$ of the population, with the largest toenails being the most affected. Related conditions range from DM and systemic hypertension to obesity among the three main pathologies ${ }^{25}$. In another study conducted at the Department of Dermatology, Venereology and Allergy of the Medical University of Wroclaw 
in Poland, a sample of 198 patients aged $\geq 65$ years was analyzed, in which a direct association was found with other comorbidities such as DM, atherosclerosis of the lower limbs and gout. In both studies, it was predominantly found in males, and the main isolated microorganism was Trichophyton rubrum, which coincides with global statistics ${ }^{27-29}$.

\section{Nail psoriasis}

It affects between $80 \%$ and $90 \%$ of patients with psoriasis, and only $5 \%$ of elderly patients have nail psoriasis without skin involvement. In these patients, the association with psoriatic arthritis involving the distal interphalangeal joints with the presence of sausage fingers (dactylitis) must be clinically differentiated from degenerative osteoarthritis ${ }^{26}$. The clinical data presented in nail psoriasis depend on the anatomical portion in which the inflammation occurs. The main inflammations are the thimble sign or "pitting" that represents parakeratosis (abnormal keratinization) in the proximal matrix, the oil-like stain that appears when the nail bed is affected, onycholysis (detachment of the nail plate) accompanied by subungual hyperkeratosis corresponds to a hyponychium condition. The chronicity of the inflammation leads to thickening of the nail plate (pachyonychia) and changes in coloration (xanthonychia). These clinical changes are indistinguishable from dermatophyte infections (onychomycosis), which requires PAS staining to reach a differential diagnosis or rule out concomitant infections ${ }^{26,30}$.

Treatment is based on the prevention of the damage caused by the injury, by wearing proper shoes, treating biomechanical alterations of the foot through orthotic devices, performing adequate, meticulous and gentle nail trimming by caregivers and/or family members and treating the underlying cause as in the case of systemic diseases. In the case of onychomycosis, various antifungals are available in the Mexican market that is administered in various routes. All of them present different success rates ranging from $60 \%$ to $80 \%$ in fungal infection cure ${ }^{27}$. It is important to emphasize the drug interactions, such as fluconazole and itraconazole, as both inhibit the P450 3A4 isoenzyme, which increases the levels of warfarin and other drugs metabolized by this route. Although terbinafine is an inhibitor of the CYP2D6 isoenzyme, it is excreted by the kidneys and induces anorexia and dysgeusia, so the risk-benefit ratio of the treatment with systemic antifungals should be assessed ${ }^{11,14,31}$. For its part, nail psoriasis represents a therapeutic challenge as most treatments are systemically based on immunomodulators such as cyclosporine and methotrexate, both with several adverse effects and drug interactions. An example is methotrexate, whose hepatic metabolism limits its use to patients without liver diseases or concomitant use of drugs through this metabolic pathway. In addition to creatinine clearance $<60 \mathrm{ml} / \mathrm{min}$, it increases toxicity risk. This is similar to what happens with cyclosporine, whose hepatic metabolism depends on CYP3A3/3A4, its excretion is biliary and renal, and it produces increased digoxin levels. Hence, systemic therapies are not recommended in most cases. However, there are multiple therapeutic options such as the use of ciclopirox olamine and amorolfine nail lacquers, practically without adverse effects but with limited effectiveness, especially in extensive forms that affect the matrix or chemical avulsion of the nail plate through the use of bifonazole/urea. Treatment of nail psoriasis refers to the use of high-potency steroids, Vitamin D analogs, topical retinoids, and phototherapy in refractory cases. Chronicity of these conditions, the poor response in the short term and difficulties regarding its application by patients make room for frequent abandonment, leading to therapeutic failures and increased resistance to antifungals as applicable ${ }^{28}$.

\section{Bullous diseases}

Bullous diseases are a heterogeneous group of autoimmune diseases that present the formation of fluid-filled lesions ranging from a few millimeters (vesicles) to several centimeters (blisters), they occur in multiple clinical contexts such as autoimmunity, drugs, trauma, infections, and genetic disorders. Within the aging process, there is a series of phenomena at the cutaneous level and in the immune system, which diminishes and alters the response to certain stimuli. Although the specific mechanism that leads to the formation of autoantibodies has not yet been elucidated ${ }^{32}$. There are several bullous diseases with different clinical-pathological features, prognosis, and treatment. In this case, we will focus on bullous pemphigoid, whose incidence and preference for a certain age group corresponds to the present study.

\section{Bullous pemphigoid}

It is the most common bullous disease in elderly patients $^{33}$ in Europe, where the elderly population is showing a steady growth due to higher life expectancy rates. Incidences of 12.5-66 cases per million inhabitants are 
reported ${ }^{32}$. A study conducted by the Department of Dermatology of Ankara Numune Educational and Research Hospital in Ankara, Turkey, from 1999 to 2003 in 4099 patients, showed the prevalence of bullous diseases within the first three dermatoses in old age ${ }^{19}$. Clinically, it is characterized initially by presenting intensely pruritic urticarial plaques, which evolve to herpetic-like vesicles developing into fluid-filled blisters that are negative for Asboe-Hansen and Nikolsky signs, which lead to erythematous exulcerations when they burst. They generally re-epithelialize after a few weeks leaving residual hyperchromic spots. The usual topography affects the upper limbs and trunk at the level of the back and abdomen, although it can affect any part of the body, rarely affecting the oral mucosa ${ }^{32,33}$. There are some risk factors involved, such as the use of antihypertensive drugs (calcium antagonists), diuretics, D-penicillamine, and dipeptylpeptidase-IV inhibitors. Some studies associate neurological disorders in up to $50 \%$ of patients ${ }^{32}$. The $\lg \mathrm{G}$ antibodies directed against two antigens currently identified as BPag1 (230KDa or collagen XVII) and BPag2 (180KDa) are two normal components of the basement membrane that is responsible for the formation of the blister at the subepidermal level, which explains why they are tense blisters. In histopathological terms, a subepidermal blister with an infiltrate is observed, with a predominance of eosinophils. Direct immunofluorescence is deemed the "gold standard" diagnosis and shows IgG and C3 deposits along the dermoepidermal junction confirming the diagnosis $^{32,33}$. There are several techniques available such as the measurement of circulating antibodies (indirect immunofluorescence) by means of ELISA or Western Blot with a sensitivity that ranges from 60 to $80 \%$. However, due to their costs, they are rarely used in our midst ${ }^{34}$.

Treatment is based on immunosuppressants such as high-potency steroids, topical for minor cases such as clobetasol propionate; systemic steroids are preferred for major cases at doses of $0.75 \mathrm{mg} / \mathrm{kg} /$ day- $1.25 \mathrm{mg} /$ $\mathrm{kg} /$ day prednisone. Some antibiotics are used for their anti-inflammatory effect in pemphigoids that are difficult to control. Their mechanism of action is uncertain, although they are believed to act at different levels of eosinophil chemotaxis such as dapsone and some others such as tetracyclines and macrolides. Azathioprine and methotrexate are used concomitantly with systemic steroids to decrease the maintenance dose of the latter. Their main adverse effects are myelosuppression and gastrointestinal discomfort. While other drugs such as cyclosporine and mycophenolate mofetil have shown good results in the treatment of this entity, other therapies such as plasmapheresis decrease steroid requirements and relapses in the short term. At present, biologic drugs such as rituximab and omalizumab have shown complete remissions in follow-ups at 23 months. However, the number of adverse effects and their cost restrict their use ${ }^{32,33}$.

The association of comorbidities, polypharmacy and reduced response in this group of patients makes the prognosis of this disease uncertain to a certain extent, which is why clinicians must make an accurate and expeditious diagnosis to minimize complications and thus directly prevent the morbidity and mortality of these patients.

\section{Malignant neoplasms}

The prevalence of non-melanoma skin cancer increases every year, due to the increase in life expectancy associated with photoaging and photocarcinogenesis. Basal cell carcinoma and squamous cell carcinoma are the two main ones, both lesions are found within epidermal tumors with relatively low metastatic capacity, and although most do not represent a risk to life, most clinical guidelines recommend surgical excision with wide margins to reduce complications such as ulceration, bleeding, expansion, and infection ${ }^{35}$. A recent article published in the BJD based on the research of two expert groups in the management of non-melanoma skin cancer proposes the evaluation of important points before making a therapeutic decision: limited life expectancy, therapeutic targets, cognitive deterioration, disability for granting consent, comorbidities, risks, complications, costs, etc. ${ }^{36}$. The objective of this review is not to detail the clinical and histological characteristics or molecular pathogenesis of these neoplasms, but to assess the risks and benefits granted to a fragile patient where risk factors are added to the detriment of their integrity. Sometimes these elderly patients, who lack the ability to decide on their own, should be treated like any other patient taking into account the ethical aspects of the therapy used ${ }^{37}$.

\section{Basal cell carcinoma}

It is the most frequently reported skin cancer, with an incidence ranging from 600,000 to 900,000 new cases per year. It is more common in men with lighter phototypes; it is considered an adulthood tumor affecting predominantly the elderly patients. It mainly affects photo-exposed areas such as the scalp, face, neck, 
and extremities, although it can occur in other topographies. Clinically, it presents itself as nodular, exophytic, neoformations of color similar to the human skin with a typical pearly border. These neoformations tend to ulcerate, presenting punch-like lesions that were formerly known as ulcus rodens ${ }^{6,8}$. The diagnosis is mainly clinical, which can help the dermatoscopy by increasing diagnostic certainty in $90 \%$, with a specificity of $89 \%$. These characteristics are arborizing vessels, ulceration, pigmented structures similar to "maple leaves," and ovoid tumor aggregates that are observed as ovoid nests or grayish blue globules, and radiated areas corresponding to pigmented tumor strands ${ }^{38}$. There are five clinical varieties; nodular/ulcerative (45$60 \%$ ), infiltrating/morpheaform (4-17\%), superficial/multifocal (15-35\%), pigmented (1-7\%), and Pinkus fibroepithelioma. In histopathological terms, they are characterized by proliferations that break off from the epidermis, forming masses and/or strands made up of basal-like cells, in a random arrangement and with a cluster of cells in the periphery, as a lattice, surrounded by clear spaces of stromal retraction. The tumor cells have a hyperchromatic nucleus with relatively small and poorly defined cytoplasm. There are numerous mitotic figures, sometimes atypical, and a quite high number of cells in apoptosis ${ }^{39}$. The pathogenesis is based on damage at the level of tumor suppressor genes such as p53, PTCH1 GLI1, GLI2, and BAX, which leads to tumor development and proliferation ${ }^{39}$.

Treatment consists of surgical excision by Mohs surgery, taking into account aggressive histological varieties, high-risk areas (H-zone of the face) with survival rates higher than $90 \%$. Other therapies such as cryosurgery and electrodessication with curettage achieve equally significant success rates. Imiquimod, 5-fluorouracil, photodynamic therapy, radiotherapy, and vismodegib are reserved for special situations in which, due to the disease stage and metastasis, topography, comorbidities or rejection to surgical treatment cannot be used $^{40}$.

\section{Squamous cell carcinoma}

It is the second most frequent skin cancer with an incidence that goes from 3\% to $10 \%$ per year. In most cases, it develops mainly because of sun exposure, which is why it is predominant in photo-exposed areas. Although it can appear in other topographies such as perianal, subungual region in related to HPV 16, 18, 33, 39,52 , and 67 , upper and lower limbs, due to previous scars, venous ulcers, and chronic inflammation situations as seen in Marjolin's ulcer that develops into wounds. Other risk factors include accumulated ultraviolet radiation (increased incidence in the tropics and decreased ozone layer), a history of sunburns, smoking, photochemotherapy, exposure to arsenic and aromatic hydrocarbons, HPV infections, immunosuppression, genetics (verrucous epidermodysplasia and xeroderma pigmentosum), ulcers and chronic fistulas, and in general all the lesions that cause chronic inflammation of the epithelium ${ }^{6,39}$. The usual topography occurs in the head and neck, affecting men more. It may arise from precursor lesions such as actinic keratosis, Bowenoid papulosis, and Bowen's disease, which are considered by some authors as true intraepidermal tumors $^{6,39}$. It appears as well delimited and generally asymptomatic erythematous patches or plaques of slow and centrifugal growth. Diagnosis is clinical, based on dermatoscopy criteria on the vascular structures presenting fork-shaped vessels of irregular shape and distribution, dotted and/or linear vessels on a whitish background, with keratin scales or scabs located in the central portion ${ }^{38}$. Histologically, it is divided into five types; common, fusiform, acantholytic, and warty cells. Classically, a proliferation is observed that comes off the epidermis, constituted by epithelial cells of atypical pleomorphic nuclei, variable mitotic rates, corneous pearls, and dyskeratosis. The tumor surface is nearly always hyperkeratotic with different degrees of parakeratosis; inflammation is observed in ulcerated tumors, composed of lymphocytes, plasma cells, neutrophils, and mononuclear infiltrate arranged in dense layers ${ }^{39}$.

Tumors greater than $2 \mathrm{~cm}$ are twice more likely to recur and metastasize, placing them within high-risk tumors together with perineural invasion (present in $2.4-14 \%$ of SCCs). Five-year survival rates are less in tumors larger than $2 \mathrm{~cm}$ that are poorly differentiated, associated with regional lymphadenopathy. Nerves $\mathrm{VI}$ and VII are frequently affected; the survival rate in these patients is $30 \%$. Metastases are more frequent in poorly differentiated SCCs. Its dissemination is directly related to the expression of metalloproteinase A2, more frequent in lymph nodes in $85 \%$ of cases, although it can spread into the lungs, mediastinum, brain, and liver. In post-transplanted patients, the metastatic risk is up to 65 times more ${ }^{41,42}$.

Treatment is based on surgical extirpation by Mohs surgery, taking into account the risk factors for a highrisk SCC. Tumor staging must be detected by biopsy, imaging studies, sentinel lymph node biopsy in case of suspicion and evidence by metastasis imaging. Although some authors only do it with the presence of high 
risk criteria. Radiotherapy and chemotherapy are reserved for unresectable cases and elderly patients ${ }^{42}$.

\section{Conclusion}

Due to the elderly population growth in Mexico, it is important to foster knowledge in multiple medical areas focused on geriatrics, mainly those with the highest morbidity and mortality rates with the dermatological area being one of them. This must be done by means of prevention strategies based on update and multidisciplinary management of elderly patients, since primary care. It is too early to say that "Dermatogeriatrics" should be implemented as a high specialty branch based on other health demands of elderly population. However, we believe that the implementation of the topic in geriatrics, Internal Medicine, Family Medicine, and Dermatology should reduce the burden on health-care services as well as reduce morbidity and mortality rates, improve the quality of life, and reduce the complications associated with the diseases that affect this age group.

\section{Ethical disclosures}

Protection of human and animal subjects. The authors declare that no experiments were performed on humans or animals for this study.

Confidentiality of data. The authors declare that no patient data appear in this article.

Right to privacy and informed consent. The authors declare that no patient data appear in this article.

\section{References}

1. Instituto Nacional de Estadistica y Geografia. Censo de Población y Vivienda; 2010. Índice de Envejecimiento; 1990-2010. Available from: http://www3.inegi.org.mx/sistemas/temas/default.aspx?s=est\&c=17484.

2. Organización Mundial de la Salud, Propuesta de Definición de Trabajo de una Persona Mayor en África Para el Proyecto MDS. Available from: http://www.who.int/healthinfo/survey/ageingdefnolder/en.

3. Secretaria de Salud. Guía de Práctica Clínica para la Valoración Geronto-Geriátrica Integral en el Adulto Mayor Ambulatorio. México: Secretaria de Salud; 2011

4. Wong JW, Koo JY. Dermatogeriatrics: a case for developing a new dermatology subspecialty. J Dermatolog Treat. 2013;24:324-6.

5. Bikle DD. Extraskeletal actions of Vitamin D. Ann N Y Acad Sci. 2016;1376:29-52.

6. Bolognia J, Jorizzo J, Schaffer J. Dermatology. In: anatomía y Fisiología. $3^{\text {rd }}$ ed., Ch. 1. Estados Unidos de América: Elsevier Saunders; 2012. p. 43-54.

7. Academia Nacional de Medicina. Dermatología geriátrica. Principales alteraciones fisiopatológicas y dermatosis más comunes en el viejo. Bol Inform Clín Ter. 2013;22:1-16.

8. Jafferany M, Huynh TV, Silverman MA, Zaidi Z. Geriatric dermatoses: a clinical review of skin diseases in an aging population. Int $\mathrm{J}$ Dermatol. 2012;51:509-22.

9. Verma SB. Dermatology for the elderly: an Indian perspective. Clin Dermatol. 2011;29:91-6.

10. Reszke R, Pełka D, Walasek A, Machaj Z, Reich A. Skin disorders in elderly subjects. Int J Dermatol. 2015;54:e332-8.
11. Norman R. Geriatric dermatology. Dermatol Ther. 2003;16:260-8.

12. Valdes-Rodriguez R, Stull C, Yosipovitch G. Chronic pruritus in the elderly: pathophysiology, diagnosis and management. Drugs Aging. 2015; 32:201-15.

13. Nair PA, Vora R. Association of systemic diseases with cutaneous dermatosis in elderly population: preliminary observation at a rural tertiary care centre. J Family Med Prim Care. 2015;4:74-8.

14. Endo JO, Wong JW, Norman RA, Chang AL. Geriatric dermatology: part I. Geriatric pharmacology for the dermatologist. J Am Acad Dermatol. 2013;68:521.e10

15. Tanei R, Hasegawa Y. Atopic dermatitis in older adults: a viewpoint from geriatric dermatology. Geriatr Gerontol Int. 2016;16 Suppl 1:75-86.

16. Kaya G, Saurat JH. Dermatoporosis: a chronic cutaneous insufficiency/ fragility syndrome. Clinicopathological features, mechanisms, prevention and potential treatments. Dermatology. 2007;215:284-94.

17. Kaya G. New therapeutic targets in dermatoporosis. J Nutr Health Aging. 2012;16:285-8.

18. Palomar F, Fornes B, Arantón L, Rumbo JM. Envejecimiento cutáneo y dermatoporosis. Enferm Dermatol. 2013;18-19:8-13.

19. Yalçin B, Tamer E, Toy GG, Ozta冈 P, Hayran M, Alli N, et al. The prevalence of skin diseases in the elderly: analysis of 4099 geriatric patients. Int J Dermatol. 2006;45:672-6.

20. Calonje JE, Brenn T, Lazar AJ, McKee PH. McKee's Pathology of the Skin. $4^{\text {th }}$ ed., Vol. 2. Edinburgh: Elsevier Saunders; 2012. p. 1150-217.

21. Choi W, Yin L, Smuda C, Batzer J, Hearing VJ, Kolbe L, et al. Molecular and histological characterization of age spots. Exp Dermatol. 2017;26: 242-8.

22. Zalaudek I, Lallas A, Longo C, Moscarella E, Tiodorovic-Zivkovic D, Ricci C, et al. Problematic lesions in the elderly. Dermatol Clin. 2013;31:549-64, 7-8.

23. Sezer E, Erbil H, Kurumlu Z, Ta冈tan HB, Etikan I. A comparative study of focal medium-depth chemical peel versus cryosurgery for the treatment of solar lentigo. Eur J Dermatol. 2007:17:26-9.

24. Ruiz A, Venegas R, Espinosa A. Dermatoscopia de las queratosis seborreicas y sus diferentes caras. Dermatol Rev Mex. 2012;56:193-200.

25. Vachiramon V, Panmanee W, Techapichetvanich T, Chanprapaph K. Comparison of $\mathrm{Q}$-switched nd: yag laser and fractional carbon dioxide laser for the treatment of solar lentigines in asians. Lasers Surg Med. 2016;48:354-9.

26. Martin B. Histopatología de la uña. Actas Dermosifiliogr. 2013;104: 564-78.

27. Araiza-Santibañez J, Tirado-Sánchez A, González-Rodríguez AL, Vázquez-Escorcia L, Ponce-Olivera RM, Bonifaz A, et al. Onychomycosis in the elderly. A 2-year retrospective study of 138 cases. Rev Med Hosp Gen Méx. 2015;79:5-10.

28. Murdan S. Nail disorders in older people, and aspects of their pharmaceutical treatment. Int J Pharm. 2016:512:405-11.

29. Arenas R, Bonifaz A, Padilla MC, Arce M, Atoche C, Barba J, et al. Onychomycosis. A Mexican survey. Eur J Dermatol. 2010;20:611-4.

30. Potts GA, Hurley MY. Psoriasis in the geriatric population. Clin Geriatr Med. 2013;29:373-95.

31. Kratzsch D, Treudler R. Dermatologic therapy in geriatric patients. J Dtsch Dermatol Ges. 2014:12:653-63.

32. Kim M, Borradori L, Murrell DF. Autoimmune blistering diseases in the elderly: clinical presentations and management. Drugs Aging. 2016;33: 711-23.

33. Tirado-Sánchez A, Díaz-Molina V, Ponce-Olivera RM. Efficacy and safety of azathioprine and dapsone as an adjuvant in the treatment of bullous pemphigoid. Allergol Immunopathol (Madr). 2012;40:152-5.

34. Zhao CY, Murrell DF. Advances in understanding and managing bullous pemphigoid. F1000Res. 2015;4:F1000.

35. Na CR, Wang S, Kirsner RS, Federman DG. Elderly adults and skin disorders: common problems for nondermatologists. South Med J. 2012; 105:600-6.

36. Schofield JK, Linos E, Callander J. Management of skin cancer in the frail elderly: time for a rethink? Br J Dermatol. 2016;175:855-6.

37. Fontanella D, Grant-Kels JM, Patel T, Norman R. Ethical issues in geriatric dermatology. Clin Dermatol. 2012;30:511-5.

38. Martín JM, Bella-Navarro R, Jordá E. Vascularización en dermatoscopia. Actas Dermosifiliogr. 2012;103:357-75.

39. Calonje JE, Brenn T, Lazar AJ, McKee PH. McKee's Pathology of the Skin. $4^{\text {th }}$ ed., Vol. 2. Edinburgh: Elsevier Saunders; 2012. p. 1076-147.

40. Wiznia LE, Federman DG. Treatment of basal cell carcinoma in the elderly: what nondermatologists need to know. Am J Med. 2016;129:655-60.

41. Díaz-Corpas T, Morales-Suárez-Varela M, Fontestad NR, A. Fuertes Próspera A, Marquina-Vila A, Jordá-Cuevas E. Carcinoma epidermoide cutáneo: definición de sus características clínico-patológicas y factores de riesgo asociados en un estudio observacional de 118 pacientes. Actas Dermosifiliogr. 2015;106:806-15.

42. Nuño-González A, Vicente-Martína FJ, Pinedo-Moraleda F, López-Estebaranz JL. Carcinoma epidermoide cutáneo de alto riesgo. Actas Dermosifiliogr. 2012;103:567-78. 